\title{
Statistical hyperbolicity in groups
}

\author{
MOON DUCHIN \\ SAMUEL LELIÈVRE \\ CHRISTOPHER MOONEY
}

\begin{abstract}
In this paper, we introduce a geometric statistic called the sprawl of a group with respect to a generating set, based on the average distance in the word metric between pairs of words of equal length. The sprawl quantifies a certain obstruction to hyperbolicity. Group presentations with maximum sprawl (ie without this obstruction) are called statistically hyperbolic. We first relate sprawl to curvature and show that nonelementary hyperbolic groups are statistically hyperbolic, then give some results for products and for certain solvable groups. In free abelian groups, the word metrics are asymptotic to norms induced by convex polytopes, causing several kinds of group invariants to reduce to problems in convex geometry. We present some calculations and conjectures concerning the values taken by the sprawl statistic for the group $\mathbb{Z}^{d}$ as the generators vary, by studying the space $\mathbb{R}^{d}$ with various norms.
\end{abstract}

20F65; 11H06, 57S30, 52A40

\section{Introduction}

We will define and study a new geometric statistic for groups in this paper, called the sprawl of a group (with respect to a generating set). Sprawl measures the average distance between pairs of points on the spheres in the word metric, normalized by the radius, as the spheres get large. This gives a numerical measure of the asymptotic shape of spheres that can be studied for arbitrary finitely generated groups and locally finite graphs.

To be precise, let

$$
E(G, S):=\lim _{n \rightarrow \infty} \frac{1}{\left|S_{n}\right|^{2}} \sum_{x, y \in S_{n}} \frac{1}{n} d(x, y),
$$

provided this limit exists. Note that since $0 \leq d(x, y) \leq 2 n$, the value is always between 0 and 2. By way of interpretation, note that $E=2$ means that one can almost always pass through the origin when traveling between any two points on the sphere without taking a significant detour. (The name is intended to invoke urban sprawl: a 
higher value means a lack of significant shortcuts between points on the periphery of the "city.")

As we will see, this statistic is not quasi-isometry invariant but nonetheless captures interesting features of the large-scale geometry, developed below in Section 2. Sprawl has connections to other geometric statistics such as divergence, almost-convexity and coarse Ricci curvature. After explaining why this statistic detects curvature properties, we show below that non-elementary hyperbolic groups always have $E(G, S)=2$ for any generating set, so we can think of $2-E$ as quantifying an obstruction to hyperbolicity in groups. We give some results about sprawl for non-hyperbolic groups, including product groups and some solvable examples (lamplighter groups).

\section{Free abelian groups and convex geometry}

For free abelian groups, there are precise results on the distribution of long words that allow spherical averaging. As we will review below in Section 3, the word metrics on $\mathbb{Z}^{d}$ are close at large scale to certain norms and the points of the spheres are distributed in a way that tends to a limit measure on the unit sphere in the norm. This allows us to replace an asymptotic computation for long words in groups by a finite computation: integrating over a polytope against an appropriate measure. Though we can compute sprawl exactly for any finite presentation of $\mathbb{Z}^{d}$, it is still an interesting problem to find the extremal values over all generating sets. That is, we are studying a group statistic that depends on the choice of generators, but how much can it vary? This becomes a (possibly hard) problem in convex geometry, which we will study below.

Definition 1 A convex body is a convex set in $\mathbb{R}^{d}$ with interior. A perimeter is the boundary of a centrally symmetric convex body in $\mathbb{R}^{d}$.

(To emphasize this point: we are using the word "perimeter" in a special way, which includes the assumption of central symmetry. Accordingly, we assume that our generating sets $S$ for $\mathbb{Z}^{d}$ are symmetric, so that $S=-S$.)

We will show that a generating set $S$ for $\mathbb{Z}^{d}$ induces a perimeter $L$ in a very simple way ( $L$ is just the boundary of the convex hull of $S$ in $\mathbb{R}^{d}$ ) and that the sprawl $E\left(\mathbb{Z}^{d}, S\right)=E(L)$ depends only on $L$. Furthermore $E(L)=E(T L)$ for linear transformations $T$, so sprawl gives an affine geometric invariant: average distance between two points on the perimeter, where both the distance and the measure have natural intrinsic definitions with respect to the shape. We conjecture that the cube and the sphere are the extreme shapes in every dimension, and we give some rigorous evidence and some numerical evidence for that below. This would mean, for example, 
that although the value for $0 \leq E \leq 2$ does vary with choice of generators, it is somewhat tightly pinched for some groups: for instance, for $G=\mathbb{Z}^{2}$, the conjecture would imply that $1.27<E<1.34$.

\section{Acknowledgments}

We thank Alex Eskin, Ralf Spatzier, Greg Kuperberg and Keith Ball. The first author is partially supported by NSF grant DMS-0906086, the second author is partially supported by ANR grants 06-BLAN-0038 and Project Modunombres, and the third author is partially supported by NSF grant RTG-0602191. We also gratefully acknowledge the helpful suggestions from anonymous referees.

\section{Hyperbolic groups and statistically hyperbolic groups}

In a graph, let us adopt the convention that for a real number $r \geq 0$, the notation $S_{r}$ denotes the metric sphere of radius $\lfloor r\rfloor$. We will study the Cayley graph as the metric model of a group, adopting the convention that the points of our metric space are the vertices (that is, the elements of the group), endowed with the distance induced by the edges (the word metric). We will write $\beta(r):=\# B_{r}(e)$ to denote the number of group elements in the closed ball of radius $r$ about the identity (or by translation-invariance, about any other center) in the group.

\subsection{Hyperbolicity}

Intuitively, we would expect maximal sprawl $(E=2)$ in spaces where geodesics diverge quickly, so that their time- $r$ points are far apart. For a geometric group theorist, the first place to look for this phenomenon would be in hyperbolic groups. Recall that a geodesic metric space is called $\delta$-hyperbolic (or just hyperbolic, without specifying a value $\delta$ ) if every geodesic triangle has the property that each side is contained in the $\delta$-neighborhood of the union of the other two sides. In such a space, suppose two geodesic rays share a common endpoint. Then if they become separated by $2 \delta$ at time $t_{0}$, they must subsequently diverge completely: the two subrays after this separation can be concatenated to form a complete quasigeodesic, because for $r>t_{0}$, any geodesic segment connecting $\gamma_{1}(r)$ and $\gamma_{2}(r)$ must return to a $2 \delta$-neighborhood of $\gamma_{i}\left(t_{0}\right)$. This means that the distance between $\gamma_{1}(r)$ and $\gamma_{2}(r)$ is at least $2 r-2\left(t_{0}+\delta\right)$. Since we have strong estimates on the distance after the rays stop fellow-traveling, our task for hyperbolic groups will be to get quantitative control of the fellow-traveling.

To illustrate the issues involved in finding the sprawl of a group, first consider the free (nonabelian) group $F_{2}$ with its standard generating set. (Here and from now on, std 
will denote the standard generating set for a group). The Cayley graph is a 4-regular tree and, to evaluate the average on the sphere directly, one forms a finite sum by fixing one point on the sphere and then counting the other points of $S_{n}$ at various distances from the first:

$$
\sum_{y \in S_{n}} d(x, y)=\frac{3}{4}(2 n)+\frac{1}{4} \frac{2}{3}(2 n-2)+\frac{1}{4} \frac{1}{3} \frac{2}{3}(2 n-4)+\frac{1}{4} \frac{1}{3} \frac{1}{3} \frac{2}{3}(2 n-6)+\cdots+0 .
$$

As $n \rightarrow \infty$, we can evaluate the sum and verify that $E\left(F_{2}\right.$, std $)=2$. This argument, however, is sensitive to the choice of generating set. What would happen for some other generating set? Does the $\delta$-hyperbolicity of the model space suffice? The answer is "No" in general. One can easily construct trees with sprawl any number between 0 and 2, trees where sprawl does not exist and trees where sprawl depends on basepoint. These trees are highly nonhomogeneous and are not quasi-isometric to any group.

Remark 2 (Sprawl and classical curvature) Moving beyond locally finite graphs and groups, we can define the sprawl for metric spaces that have natural measures on spheres. Instead of counting measure one may take Hausdorff measure in the appropriate dimension, for example. Thus for a space and measure $(X, \mu)$, we can write

$$
E(X):=\lim _{r \rightarrow \infty} \frac{1}{\mu\left(S_{r}\right)^{2}} \int_{S_{r} \times S_{r}} \frac{1}{r} d(x, y) d \mu(x) d \mu(y) .
$$

One can quickly show that the hyperbolic plane (and thus hyperbolic space of any dimension) has $E=2$ : for two rays making angle $\theta$ at their common basepoint, $d\left(\gamma_{1}(r), \gamma_{2}(r)\right) \geq 2 r-c(\theta)$, where $c(\theta)$ is a constant depending on $\theta$.

Indeed, it is not hard to identify a relationship between sprawl and curvature: if $E_{r}$ is defined to be the average distance between pairs of points on $S_{r}$ and $M_{\kappa}$ is the model space of constant sectional curvature $\kappa$, it is easily observed that for every fixed value of $r$, the values $E_{r}\left(M_{\kappa}\right)$ are strictly decreasing in $\kappa$ (taking $\kappa \leq \pi^{2} r^{2}$ so that $S_{r}$ is non-empty). Below, in Section 5, we will gather further evidence, finding that certain Banach spaces are also "arranged correctly" by sprawl: those that are closer to flat in measures coming from convex geometry have sprawl closer to $E\left(M_{0}\right)$.

However, a $\delta$-hyperbolic space, indeed even a tree, need not have $E=2$, and exponential growth of balls or spheres does not suffice. For instance, consider modifying the four-regular tree by choosing one axis and modifying the degree at each vertex in that axis as a function of distance from the origin. Examples constructed in this way can achieve all values $0 \leq E \leq 2$, and can also have $E$ not exist or depend on basepoint. Thus to prove that hyperbolic groups have maximal sprawl, it is essential to make use of the homogeneity guaranteed by a transitive group action. We will use this by appealing 
to a strong result of Michel Coornaert giving definite exponential growth (not just a growth rate but furthermore a bound on the coefficients) for hyperbolic groups.

Remark 3 (Divergence, almost-convexity, coarse Ricci curvature) We have defined the sprawl based on the distances between pairs of points $x, y \in S_{n}$, by studying the asymptotics of the average distance. At least three other geometric statistics also study the geometry of pairs of points in the sphere.

- Divergence is defined by the asymptotics of the "external distance" between $x, y \in S_{n}$ (ie length of shortest path lying outside $B_{n}$ ). This is widely studied for groups since Gromov suggested its importance, with early results by Gersten [14; 15], and Kapovich and Leeb [18]. Hyperbolicity is equivalent to exponential divergence in a certain precise sense.

- Almost-convexity for groups is defined by bounding the "internal distance" between $x, y \in S_{n}$ (ie length of shortest path inside $B_{n}$ ) in terms of the distance between $x$ and $y$. This was defined by Cannon in [4] and further explored in many papers, such as Hermiller and Meier [17], Elder and Hermiller[12], and Cleary and Taback [5]. Cannon conjectured that hyperbolic groups are almost-convex.

- Ricci curvature for manifolds is defined by considering infinitesimal spheres at a pair of basepoints, and measuring the average distance between corresponding points on the spheres. The curvature is negative if that distance is greater than the distance between basepoints, positive if it is smaller, and zero if it is equal. Coarse Ricci curvature mimics this construction in a manner usable for groups. This was defined by Yann Ollivier in [21] and closely resembles alternate definitions of Cédric Villani and coauthors (see [22]).

Thus the definition of sprawl gives it a family resemblance to other synthetic conditions carrying strong geometric information, with relationships to curvature.

Recall that a hyperbolic group is called elementary if it is finite or has a finite-index cyclic subgroup.

Theorem 4 Let $G$ be a non-elementary hyperbolic group. Then $E(G, S)=2$ for any finite generating set $S$. (That is, every presentation is statistically hyperbolic.)

Proof Recall that $z$ is said to be (metrically) between $x$ and $y$ if $d(x, z)+d(z, y)=$ $d(x, y)$. A set is between two other sets if there exists a triple of points, one from each of the sets, satisfying the betweenness condition. 
Choose any $0<\rho<1$ and $x \in S_{n}$ and let $x^{\prime}$ be an arbitrary point on $S_{\rho n}$ between $e$ and $x$. We need to bound the number of $w \in S_{n}$ such that $B_{2 \delta}\left(x^{\prime}\right)$ is between $e$ and $w$. But if $w^{\prime}$ is a point in $S_{\rho n}$ between $e$ and $w$, then $d\left(w^{\prime}, w\right)=n-\lfloor\rho n\rfloor$. That means that the number of such $w$ is overcounted by $\left|B_{2 \delta}\right| \cdot\left|S_{n-\lfloor\rho n\rfloor}\right|$.

For every point $v$ of $S_{n}$ which is not of this kind, $d(x, v) \geq 2(n-\lfloor\rho n\rfloor-\delta) \geq$ $2(n-\rho n-\delta)$ because the geodesics from the identity to $x$ and to $v$ have $2 \delta$-diverged by time $\lfloor\rho n\rfloor$. Thus,

$$
\sum_{x, y \in S_{n}} d(x, y) \geq 2(n-\rho n-\delta)\left(\left|S_{n}\right|-\left|B_{2 \delta}\right| \cdot\left|S_{n-\lfloor\rho n\rfloor}\right|\right) \cdot\left|S_{n}\right| .
$$

Now we make use of the homogeneity. Coornaert proved in [6] that for every nonelementary hyperbolic group with fixed generating set, there are bounded coefficients of exponential growth:

$$
\exists c_{1}, c_{2}>0, \omega>1 \quad \text { s.t. } \quad c_{1} \omega^{n} \leq \beta(n) \leq c_{2} \omega^{n} \quad \forall n \in \mathbb{N} .
$$

It follows from these inequalities that

$$
\frac{\left|S_{n-\lfloor\rho n\rfloor}\right|}{\left|S_{n}\right|}=\frac{\beta(n-\lfloor\rho n\rfloor)-\beta(n-\lfloor\rho n\rfloor-1)}{\beta(n)-\beta(n-1)} \rightarrow 0
$$

as $n \rightarrow \infty$, which together with $(\star)$ gives us

$$
E(G, S)=\lim _{n \rightarrow \infty} \frac{\sum d(x, y)}{n\left|S_{n}\right|^{2}} \geq 2(1-\rho) .
$$

Since $0<\rho<1$ was arbitrary, this means $E=2$.

To quickly clarify the necessity for the non-elementary hypothesis: for $G=\mathbb{Z}$ and any finite generating set, the spheres of large radius are divided into a positive part and a negative part, each of uniformly bounded diameter. Thus a pair of points has bounded distance with probability $1 / 2$ and distance boundedly close to $2 n$ with probability $1 / 2$. This gives $E(\mathbb{Z}, S)=1$ for all finite generating sets $S$.

\subsection{Some statistically hyperbolic groups and spaces}

In this section we exhibit several examples of non-hyperbolic groups with statistically hyperbolic presentations. We first consider groups that are direct products with a hyperbolic factor and then use the results on products to show that Diestel-Leader graphs, which model solvable geometry, are statistically hyperbolic.

Teichmüller space and the curve complex are also statistically hyperbolic with respect to natural measures (Dowdall, Duchin and Masur [7; 8]). On the other hand, one would 
expect for nilpotent groups not to be statistically hyperbolic; for the Heisenberg group with its standard generators, this follows from the limit measure constructed by Duchin and Mooney in [11].

In the last section we showed that $\delta$-hyperbolicity and precise bounds on growth suffice to show statistical hyperbolicity. To build on that, let us say that a based space $\left(H, h_{0}\right)$ has definite exponential growth if the growth function $\beta(n)$ of balls of radius $n$ centered at $h_{0}$ in $H$ satisfies ( $\left.\dagger\right)$. Given a sequence of finite sets $A_{n}$, we will say that almost all points of $A_{n}$ satisfy a property $(P)$, or that $(P)$ has full measure, if the proportion of $A_{n}$ satisfying $(P)$ tends to one as $n \rightarrow \infty$.

The sphere of radius $n$ in a product space $H \times K$ projects to not a sphere but to a ball in each factor; with definite exponential growth in $H$, we will see almost all of the projection in an annulus $A_{n}^{H}$. Thus we need estimates for distances when points are on spheres of different radii. We also need to know that spheres in these annuli are evenly covered by which we mean that there is a function $f_{n}: \mathbb{N} \rightarrow \mathbb{N}$ such that $\#\left(\pi^{-1}(h) \cap S_{n}^{X}\right)=f_{n}\left(|h|_{H}\right)$ for almost all $h \in A_{n}^{H}$. That is, up to measure, the size of the preimage in $X$ of $h \in A_{n}^{H}$ should only depend on the "radius" in $H$.

For the following technical lemma, the basic example is that $H$ is a direct factor of $X$ and that $\pi: X \rightarrow H$ is coordinate projection. Recall that a semi-contraction is a distance non-increasing (ie 1-Lipschitz) map.

Lemma 5 (Annulus lemma) Let $\left(X, x_{0}\right)$ and $\left(H, h_{0}\right)$ be based graphs, suppose $H$ is $\delta$-hyperbolic with definite exponential growth, and fix any $0<\rho<1$. Consider the annulus $A_{n}^{H}=\bigsqcup_{k=\rho n}^{n} S_{k}^{H}\left(h_{0}\right)$ in $H$ and the sphere $S_{n}^{X}=S_{n}^{X}\left(x_{0}\right)$ in $X$. Let $\pi:\left(X, x_{0}\right) \rightarrow\left(H, h_{0}\right)$ be a semi-contraction, mapping almost all points of $S_{n}^{X}$ into $A_{n}^{H}$ such that spheres are evenly covered. Then

$$
\liminf _{n \rightarrow \infty} \frac{1}{\left|S_{n}\right|^{2}} \sum_{x, y \in S_{n}} \frac{1}{n} d(x, y) \geq 2 \rho .
$$

This is proved by verifying that when $\rho n \leq i, j \leq n$, then the average distance between a point in $S_{i}^{H}$ and a point in $S_{j}^{H}$ is bounded below by $i+j-2 \delta-2 \rho n-c n \omega^{-\rho n}$ for a constant $c$, where $\omega$ is the growth rate of $H$, as in $(\dagger)$.

We will apply this lemma to products of the form $H \times K$ where $H$ is hyperbolic and $K$ grows strictly slower-that is, $K$ has subexponential growth, or has a smaller exponential growth rate. Let us say that a generating set for a product is split if every generator projects to the identity in one of the factors. 
Proposition 6 (Products with a dominated factor) Suppose $H$ is a non-elementary hyperbolic group, $K$ is finitely generated, and $S$ is a split finite generating set for $H \times K$ such that the growth function of $H$ dominates the growth function of $K$ with generators projected to the factors from $S$. Then $(H \times K, S)$ is a statistically hyperbolic presentation.

Proof Let $\pi$ be projection to the $H$ factor from $X=H \times K$ and note that

$$
S_{k}^{X}=\bigcup_{i=0}^{k} S_{i}^{H} \times S_{k-i}^{K} .
$$

Thus one easily verifies the hypotheses of the annulus lemma. Letting $\rho \rightarrow 1$ gives $E(X, S)=2$.

Thus, for example, $F_{2} \times \mathbb{Z}$ is statistically hyperbolic with its standard generators, even though it is not $\delta$-hyperbolic.

Another class of statistically hyperbolic spaces is the Diestel-Leader graphs. We describe them briefly here and refer the reader to Woess [23] for a more thorough treatment and some relevant properties. For $m, p \geq 2$, take an $(m+1)$-valent tree $T_{1}$ and a $(p+1)$-valent tree $T_{2}$. Choose ends and corresponding horofunctions $f_{1}$ and $f_{2}$. This gives height functions $h_{1}=-f_{1}$ and $h_{2}=f_{2}$ on the trees. We visualize $T_{1}$ as "hanging down" from its end at height $+\infty$ and $T_{2}$ as "growing up" from its end at $-\infty$. The Diestel-Leader graph $\operatorname{DL}(m, p)$ is defined to be the subspace of $T_{1} \times T_{2}$ on which $h_{1}=h_{2}$. A height function $h$ is induced on this graph from the tree factors, since their height functions match. Like Cayley graphs, Diestel-Leader graphs have vertex-transitive group actions by isometries, which guarantees that geometric invariants of $\mathrm{DL}(m, p)$ do not depend on the choice of basepoint.
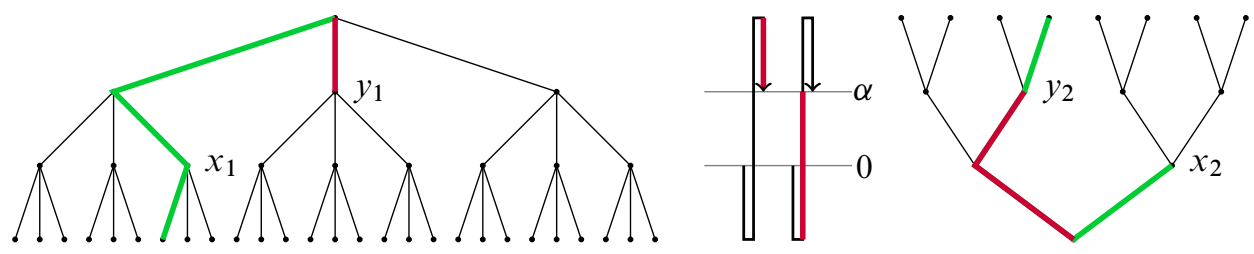

Figure 1: Here, a geodesic of length $n=5$ proceeds from $x$ to $y$ in $\operatorname{DL}(3,2)$ by following the marked paths in each tree according to the height diagram, ending at height $\alpha=1$. The $(\alpha+n) / 2$ choices that would lead to different points at the same height are shown.

These graphs are considered models for solvable geometry: the structure described above is in precise analogy with the geometry of Sol, which has hyperbolic plane factors 
in the place of trees. Eskin, Fisher and Whyte [13] exploit this analogy to completely classify Diestel-Leader graphs and spaces with Sol geometry up to quasi-isometry.

Denote the coordinate projections by $\pi_{i}: \operatorname{DL}(m, p) \rightarrow T_{i}$ for $i=1,2$. A geodesic $\gamma$ in $\operatorname{DL}(m, p)$ is said to turn if it switches from increasing in height to decreasing in height or vice versa. Geodesics in Diestel-Leader graphs have at most two turns, coming from turns in the tree factors, as in Figure 1. The following lemma tells us that spheres of large radius in a Diestel-Leader graph are "concentrated in distant heights."

Lemma 7 (Concentration in height) Let $X=\operatorname{DL}(m, p)$ be a Diestel-Leader graph, fix a basepoint $x \in X$ with $h(x)=0$ and fix any $0<\rho<1$. Then for almost all $y \in S_{n}(x)$,

$$
\rho n \leq|h(y)| \leq n
$$

Proof If $m>p$ this is clear because of the greater branching in $T_{1}$; almost all points will be at height roughly $-n$. We consider the case $m=p$ and positive ending height. The number of points at height $\alpha$ in $S_{n}=S_{n}(x)$ is on the order of $m^{(\alpha+n) / 2}$ (see Figure 1). But then we can count the proportion of points below height $\rho n$ and we find that

$$
\frac{\sum_{\alpha=0}^{\rho n} m^{(\alpha+n) / 2}}{\sum_{\alpha=0}^{n} m^{(\alpha+n) / 2}}=\frac{\sum_{\alpha=0}^{\rho n} m^{\alpha / 2}}{\sum_{\alpha=0}^{n} m^{\alpha / 2}} \rightarrow 0 \text { as } n \rightarrow \infty .
$$

Theorem 8 (Diestel-Leader graphs) For any $m, p \geq 2$, the Diestel-Leader graph $X=\mathrm{DL}(m, p)$ is statistically hyperbolic.

Proof In order to apply the Annulus Lemma, we need only verify the even covering condition for the coordinate projection $\pi_{1}: X \rightarrow T_{1}$. This amounts to fixing $x, n$ and $d_{1}=d_{T_{1}}\left(x_{1}, y_{1}\right)$, and confirming that the number of possible $y_{2}$ does not depend on $y_{1}$. We find that $y_{1}$ determines $\alpha, d_{2}$ and the lowest point visited in $T_{2}$. The number of valid choices of $y_{2}$ given $y_{1}$ is counted by traveling up from the lowest point in $T_{2}$ a total of $s:=\alpha+\frac{n-d_{1}}{2}$ steps, which can be done in either $p^{s}$ or $(p-1) \cdot p^{s-1}$ ways, depending on whether the lowest point is at height 0 or $<0$.

When $m=p$, the Diestel-Leader graph $\operatorname{DL}(m, m)$ can be realized as the Cayley graph of the lamplighter group $\mathbb{Z}_{m} \prec \mathbb{Z}$ for a certain natural generating set (or, more generally, $F \prec \mathbb{Z}$ for any finite group $F$ of order $m$ ). These interesting solvable groups are not nilpotent and they are not finitely presented.

Corollary 9 (Lamplighter groups) The lamplighter groups $\mathbb{Z}_{m} \prec \mathbb{Z}$ have statistically hyperbolic presentations. 
We know of no examples of groups of exponential growth with $E<2$, but because of the facts above it would be particularly interesting to investigate groups of non-uniform exponential growth.

\section{Reducing from free abelian groups to convex geometry}

In the free abelian groups $\mathbb{Z}^{d}$, studying the large-scale metric geometry is greatly aided by the natural embedding in $\mathbb{R}^{d}$. The (finitely generated) word metrics on $\mathbb{Z}^{d}$ are asymptotic to polyhedral norms on $\mathbb{R}^{d}$, so that these norms can be thought of as limit metrics coming from group theory. In fact, a stronger statement is true: the finitely generated word metrics on $\mathbb{Z}^{d}$ are within bounded distance of the limiting norms on $\mathbb{R}^{d}$ (originally due to Burago [3] and shown by an elementary geometric argument by the authors in [9]). Recall that any perimeter in $\mathbb{R}^{d}$ induces an associated norm, namely the norm for which that convex body is the unit sphere. If a generating set for $\mathbb{Z}^{d}$ is called $S$, let $|\mathrm{w}|$ denote the length of $\mathrm{w} \in \mathbb{Z}^{d}$ in the word metric and let $L$ be the boundary of the convex hull of $S$ in $\mathbb{R}^{d}$. Then, denoting the associated norm by $\|\cdot\|_{L}$, bounded difference means that there is a constant $K$ depending on $S$ such that

$$
\|\mathrm{w}\|_{L} \leq|\mathrm{w}| \leq\|\mathrm{w}\|_{L}+K
$$

for all $w \in \mathbb{Z}^{d}$. This limit shape $L$ describes the asymptotic shape of spheres in the sense that the asymptotic cone of $\left(\mathbb{Z}^{d}, S\right)$ is $\left(\mathbb{R}^{d},\|\cdot\|_{L}\right)$, or simply in the sense that $\frac{1}{n} S_{n} \rightarrow L$ in $\mathbb{R}^{d}$ as a Gromov-Hausdorff limit.

In the paper [9], we proved counting results for spheres in word metrics on $\mathbb{Z}^{d}$, showing that counting measure on the discrete spheres $S_{n}$ converges to the cone measure $\mu_{L}$ on $L$, as pictured in Figure 2. The case of that theorem that is useful for us here states
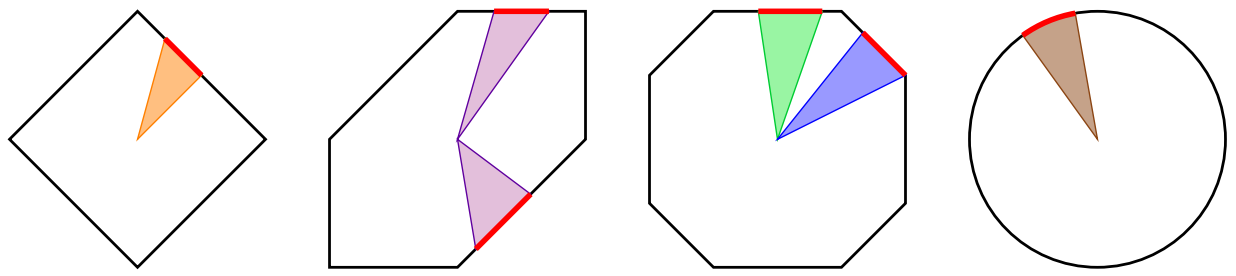

Figure 2: Six arcs are shown inside different perimeters $L_{i}$, each arc having cone measure $\mu_{L_{i}}(A)=1 / 14$.

that

$$
\lim _{n \rightarrow \infty} \frac{1}{\left|S_{n}\right|^{2}} \sum_{\mathrm{x}, \mathrm{y} \in S_{n}} \frac{1}{n} d(\mathrm{x}, \mathrm{y})=\int_{L \times L}\|\mathrm{x}-\mathrm{y}\|_{L} d \mu_{L}(\mathrm{x}) d \mu_{L}(\mathrm{y})
$$


(The original theorem addresses more general averaging problems.) Thus it follows immediately that

$$
E\left(\mathbb{Z}^{d}, S\right)=\int_{L \times L}\|\mathrm{x}-\mathrm{y}\|_{L} d \mu_{L}(\mathrm{x}) d \mu_{L}(\mathrm{y}) .
$$

for all finite generating sets $S$.

We can define the sprawl of any perimeter $L$ by the right-hand side, which we can denote by $E(L)$, measuring average distance between points of $L$ in its intrinsic geometry. We remark that $E(L)=E(T L)$ for any linear transformation $T: \mathbb{R}^{d} \rightarrow \mathbb{R}^{d}$, since both the norm and the measure push forward under linear transformation. That is, $\|T \times-T \mathrm{y}\|_{T \mathrm{~L}}=\|\mathrm{x}-\mathrm{y}\|_{L}$ and $d \mu_{T \mathrm{~L}}(T \times)=d \mu_{L}(\mathrm{x})$. That means in particular that the intrinsic geometry comes with a natural scale.

One immediate consequence of the reduction to convex geometry is a lower bound on $E\left(\mathbb{Z}^{d}, S\right)$.

Proposition $10 E(L)>\frac{1}{2}$ for all perimeters $L$ in $\mathbb{R}^{d}$.

Proof Fix an arbitrary point $x \in L$ and let $Q$ be the convex hull of $L$. The points of $L$ whose distance from $x$ is less than one are those contained in $Q+x$, the translated copy of $Q$ centered at $x$. Since $L+x$ contains 0 and $Q$ is convex, there is a hyperplane $P$ through 0 which does not intersect the interior of $Q+x$. So the interior of $Q+x$ is on one side of $P$ and, by central symmetry, half of the cone measure lies on each side of $P$. Thus the average distance on $L$ from $x$ is $\geq(1 / 2)(1)$. To obtain the strict inequality, just note that the distance from $x$ to $-x$ is always 2 and so a small neighborhood of $-x$ contributes an amount near 2 to the average.

\section{Values of sprawl for normed spaces}

In this section we describe the results of some calculations to explore the values taken by sprawl in the normed spaces $\left(\mathbb{R}^{d},\|\cdot\|_{L}\right)$. We refer readers to a short companion paper [10] where an algorithm for computing the sprawl is presented and the numerical data is further developed.

\subsection{Polygonal norms in the plane}

Example 11 Let $P_{k}$ be the regular $k$-gon and let $S^{1}$ be the round circle. Then

$$
\begin{aligned}
& E\left(P_{4}\right)=\frac{4}{3} ; \\
& E\left(P_{6}\right)=\frac{23}{18}<\frac{4}{3} ;
\end{aligned}
$$




$$
\begin{aligned}
& E\left(P_{8}\right)=\frac{1+2 \sqrt{2}}{3}<\frac{23}{18} \\
& E\left(P_{x}\right)=\left\{\begin{array}{l}
\frac{4}{\pi} \cdot\left(\frac{\pi / x}{\tan (\pi / x)}+\frac{1}{3} \frac{\pi}{x} \tan \left(\frac{\pi}{x}\right)\right), \\
\frac{4}{\pi} \cdot\left(\frac{\pi / x}{\sin (\pi / x)}-\frac{1}{6} \frac{\pi}{x} \sin \left(\frac{\pi}{x}\right)\right),
\end{array},\right. \\
& E\left(S^{1}\right)=\frac{4}{\pi} .
\end{aligned}
$$

Example 11, shown in Figure 3 below, shows the nontrivial dependence of sprawl on the choice of generating set. Note that $P_{4}$ is the limit shape for $\left(\mathbb{Z}^{2}, \pm\left\{\mathrm{e}_{1}, \mathrm{e}_{2}\right\}\right)$ and $P_{6}$ is affinely equivalent to the limit shape for $\left(\mathbb{Z}^{2}, \pm\left\{\mathrm{e}_{1}, \mathrm{e}_{2}, \mathrm{e}_{1}+\mathrm{e}_{2}\right\}\right)$. Since the word metrics of a group $G$ with respect to finite generating sets $S, S^{\prime}$ are quasi-isometric, we see that sprawl is not a quasi-isometry invariant.

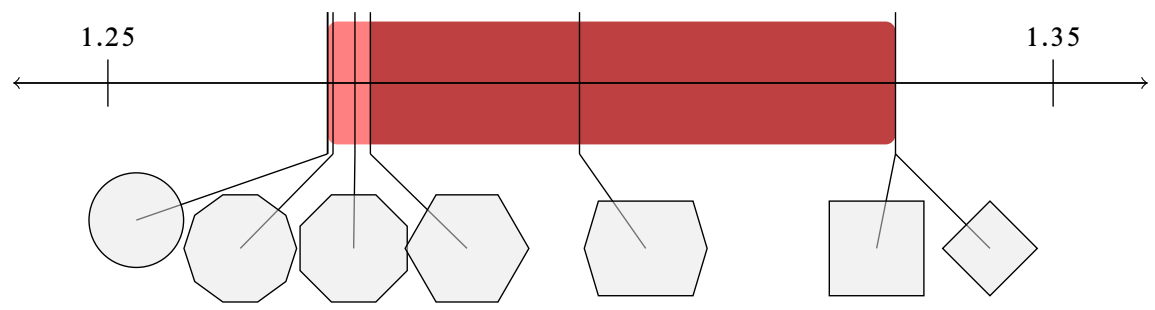

Figure 3: Sprawls of hexagons are known to range over precisely the darker interval. The other regular polygons are in the light interval, converging quickly down to the circle. Conjecturally, this is the full range of sprawls for norms on $\mathbb{R}^{2}$.

Using these facts above and a rational approximation argument, we observe a range of sprawls that can be achieved in $\mathbb{Z}^{2}$.

Proposition 12 A dense subset of the interval $\left[\frac{4}{\pi}, \frac{4}{3}\right]$ is contained in the set

$$
\left\{E\left(\mathbb{Z}^{2}, S\right): \text { gensets } S\right\} \text {. }
$$

Proof There is a continuous path $L_{t}$ through the space of perimeters that starts with the circle and ends with the square. The sprawl passes through all values from $4 / \pi$ to $4 / 3$ along the path.

Any such perimeter $L_{t}$ can be approximated arbitrarily closely by a rational polygon, which can be rescaled to an integer polygon without changing $E$. The sprawl of a polygon is continuous in the coordinates of its vertices, and $E$ of the approximants 
approaches $E$ of the original body. Finally, the set of integer vertices can be completed to a generating set without changing $E$, since the sprawl only depends on the extreme vertices.

Conjecture 13 (Sprawl Conjecture for $d=2$ ) The circle and the square are the extreme cases for all perimeters in $\mathbb{R}^{2}$. That is,

$$
\left\{E(L) \text { : perimeters } L \subset \mathbb{R}^{2}\right\}=\left[\frac{4}{\pi}, \frac{4}{3}\right] .
$$

Example 14 (Sprawls of hexagons and three-generator presentations)

$$
\{E(H) \text { : hexagons } H\}=\left[\frac{23}{18}, \frac{4}{3}\right] .
$$

Thus, $\frac{23}{18} \leq E\left(\mathbb{Z}^{2}, S\right) \leq \frac{4}{3}$ whenever $|S| \leq 6$.

This provides evidence, taken together with the fast convergence for sprawls of regular polyhedra towards $4 / \pi$, for the conjecture. Further evidence is given in the next section, where the sphere and cube are shown to be sharp bounds asymptotically as $d \rightarrow \infty$.

\subsection{Higher dimension: The not-so-flatness of $\mathbb{Z}^{d}$}

In this section, we will consider the $\ell_{2}, \ell_{\infty}$ and $\ell_{1}$ norms on $\mathbb{R}^{d}$, keeping in mind that the first is approximated and the others are realized by word metrics on $\mathbb{Z}^{d}$ at large scale. The sphere Sphere ${ }_{d} \subset \mathbb{R}^{d}$ induces the $\ell_{2}$ metric and it can be arbitrarily closely approximated by word metrics on $\mathbb{Z}^{d}$. The cube is the limit shape for $\mathbb{Z}^{d}$ with a nonstandard generating set $\left\{ \pm \mathrm{e}_{1} \cdots \pm \mathrm{e}_{d}\right\}$, and it induces the $\ell_{\infty}$ metric on $\mathbb{R}^{d}$. The orthoplex (or cross-polytope) Orth $d$ is the limit shape for $\mathbb{Z}^{d}$ with its standard generating set $\pm\left\{\mathrm{e}_{i}\right\}$, and it induces the $\ell_{1}$ metric on $\mathbb{R}^{d}$. Note that the $\ell_{1}$ norm and $\ell_{\infty}$ norm are isometric when $d=2$ but not in higher dimension.

As the dimension goes to infinity, the computation of average distance becomes quite intuitive for the sphere and the cube. Suppose $d$ is very large. For Sphere ${ }_{d}$, the round unit sphere with the standard Euclidean metric; take one point to be at the north pole without loss of generality. Then by concentration of measure, the second point is almost surely on the equator, so the distance between them is nearly $\sqrt{1^{2}+1^{2}}=\sqrt{2}$. On the other hand, for Cube ${ }_{d}$ with sup metric, the distance computation is performed by sampling the random variable $\left|x_{i}-y_{i}\right|$, which ranges between 0 and 2 , a total of $d$ times. For very large $d$, we should expect this supremum to tend to 2 . This reasoning predicts that $E\left(\right.$ Sphere $\left._{d}\right) \rightarrow \sqrt{2}$ and $E\left(\mathrm{Cube}_{d}\right) \rightarrow 2$. For the $\ell_{1}$ norm we do not know of a heuristic to predict the answer in high dimensions, but the answer turns out to take quite a simple closed form, giving the limit $E\left(\mathbb{Z}^{d}\right.$, std $) \rightarrow \frac{3}{2}$ as $d \rightarrow \infty$. 
By way of interpretation, this says that a cubical generating set gives $\mathbb{Z}^{d}$ more and more hyperbolic-like geometry as $d$ gets large, while the standard word metric is bounded uniformly away both from hyperbolic geometry and from Euclidean geometry (see Figure 4). In group theory we are accustomed to describing the group $\mathbb{Z}^{d}$ as "flat" because it is quasi-isometric to Euclidean space. However, using this statistic that gives a finer measure of large-scale curvature, we see that the standard generators give rise to a Cayley graph with significantly less efficient "shortcuts" than in Euclidean space, and that there exist generators for large $d$ which make the geometry a good deal closer to hyperbolic than flat.

The formulas for the sprawl of the sphere, cube and orthoplex are given in the following closed forms, with rates of convergence as $d \rightarrow \infty$ computed as functions of $d$. (See [10] for details.) Here $\Gamma(x)$ denotes Euler's gamma function.

\section{Example 15}

$$
\begin{aligned}
& E\left(\text { Sphere }_{d}\right)=\frac{2^{d-1}}{\sqrt{\pi}} \cdot \frac{\Gamma\left(\frac{1}{2} d\right)^{2}}{\Gamma\left(d-\frac{1}{2}\right)} \\
& ; \sqrt{2}-E\left(\text { Sphere }_{d}\right) \sim \frac{1}{8 d} \text {. } \\
& E\left(\text { Cube }_{d}\right)=\frac{2 d+2}{d}-\left[\frac{2 d+1}{2 d^{2}} \cdot \frac{4^{d} d !^{2}}{(2 d) !}\right] ; 2-E\left(\text { Cube }_{d}\right) \sim \frac{\sqrt{\pi}}{\sqrt{d}} . \\
& E\left(\operatorname{Orth}_{d}\right)=\frac{3 d-2}{2 d-1} \\
& ; \quad \frac{3}{2}-E\left(\operatorname{Orth}_{d}\right) \sim \frac{1}{4 d} .
\end{aligned}
$$

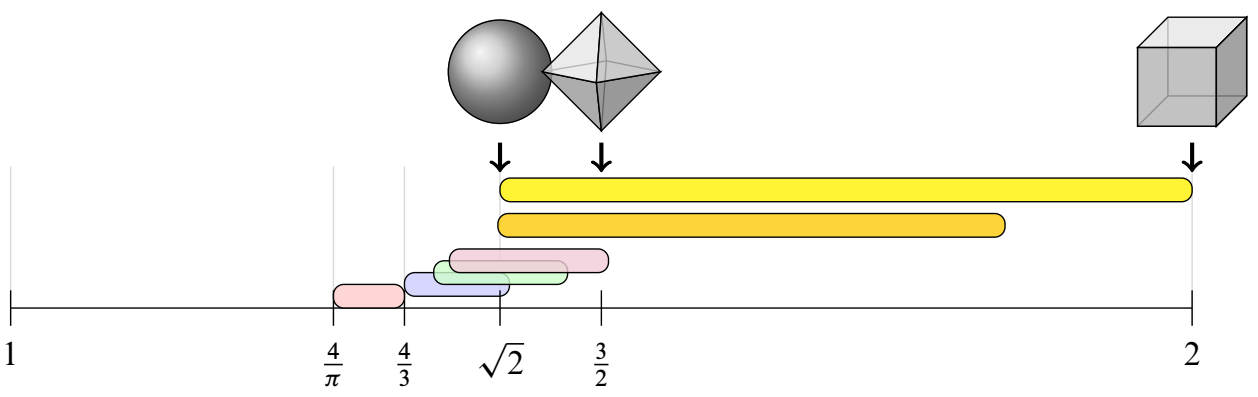

Figure 4: The range $\left[E\left(\right.\right.$ Sphere $\left._{d}\right), E\left(\right.$ Cube $\left.\left._{d}\right)\right]$ is shown for $d=2,3,4,5,100, \infty$.

\section{An affine isoperimetric conjecture}

By rational approximation of convex bodies (as in the proof of Proposition 12), we find that a dense subset of the interval $\left[E\left(\right.\right.$ Sphere $\left.\left._{d}\right), E\left(\mathrm{Cube}_{d}\right)\right]$ is contained in the set of 
values realized by groups, so

$\left[E\left(\right.\right.$ Sphere $\left._{d}\right), E\left(\right.$ Cube $\left.\left._{d}\right)\right] \subseteq \overline{\left\{E\left(\mathbb{Z}^{d}, S\right): \text { gensets } S\right\}}=\left\{E(L)\right.$ : perimeters $\left.L \subset \mathbb{R}^{d}\right\}$.

We conclude by conjecturing that this is everything.

Conjecture 16 (Sprawl Conjecture) The sphere and the cube are the extremes for the sprawl in each dimension. That is, for $d=2,3,4, \ldots$,

$$
\left\{E(L): \text { perimeters } L \subset \mathbb{R}^{d}\right\}=\left[E\left(\text { Sphere }_{d}\right), E\left(\text { Cube }_{d}\right)\right] .
$$

This conjecture would complete the description for free abelian groups of the dependence of this curvature statistic on the generating set, showing the values to be "pinched" as in Figure 4.

A similar conjecture could be formulated for the balls instead of the spheres: consider the average distance statistic for solid bodies defined by

$$
\hat{\mathrm{E}}(\Omega):=\int_{\Omega \times \Omega}\|\mathrm{x}-\mathrm{y}\|_{\Omega} d \operatorname{Vol}(x) d \operatorname{Vol}(y)
$$

for convex, centrally symmetric $\Omega \subset \mathbb{R}^{d}$, where Vol is the normalization of Lebesgue measure so that $\operatorname{Vol}(\Omega)=1$. Here, one can apply the powerful Brascamp-LiebLuttinger inequality (as in Gluskin and Milman [16, Theorem 1]), with $\lambda=(1,-1)$, to see that $\hat{E}$ is minimized by (round) balls. (This strongly suggests that average distance on a perimeter should be minimized by spheres as well, but there are fewer established techniques for dealing with perimeters than with solid bodies.)

More evidence for the Sprawl Conjecture can be found in the high-dimensional asymptotics. Because $E \leq 2$ always, it is immediate that

$$
\lim _{d \rightarrow \infty} \sup \{E(L)\}=\lim _{d \rightarrow \infty} E\left(\text { Cube }_{d}\right)=2 .
$$

Arias-de-Reyna, Ball and Villa consider $\hat{\mathrm{E}}(\Omega)$ and prove that for almost all pairs of points in $\Omega \times \Omega$, the distance is greater than $\sqrt{2}(1-\epsilon)$ [1, Theorem 1]. As they note, the points in the ball become concentrated in its boundary as $d \rightarrow \infty$. This shows that the $E\left(\right.$ Sphere $\left._{d}\right)$ is a lower bound for sprawl asymptotically, ie

$$
\lim _{d \rightarrow \infty} \inf \{E(L)\}=\lim _{d \rightarrow \infty} E\left(\text { Sphere }_{d}\right)=\sqrt{2} .
$$

Thus the Sprawl Conjecture is true asymptotically.

The classical isoperimetric problem is uniquely solved by round balls and spheres. It has an affine-invariant formulation as well and for that version the extremes among 
centrally symmetric convex bodies are realized by round balls on one end and cubes on the other. Indeed, in modern usage, the term affine isoperimetric inequality is used to describe any situation where a geometric affine invariant takes an extreme value at balls and ellipses. We have seen that average distance in a solid body satisfies an affine isoperimetric inequality. Another example is given by the volume ratio, measuring the ratio of the volume of $\Omega$ to that of an inscribed ellipsoid - among centrally symmetric convex bodies, it is clearly minimized by balls and is also known to be maximized by cubes. Other affine invariants with round balls at one extreme and cubes at the other include affine surface area and volume of polar projection body. (See Lutwak [20] for a survey.)

The finding reported in the last section that the sprawl sees the orthoplex to look more like a sphere than a cube in high dimension is in keeping with the comparisons given by other invariants. For instance, the volume ratios of Sphere $_{d}$, Orth $_{d}$, and Cube $d$ are $1, \leq 2$ and $\sim \sqrt{d} / 2$, respectively, making $\ell_{1}$ far more flat-like than $\ell_{\infty}$. A second classical question in convex geometry is to find "almost spherical sections" of Banach spaces; that is, subspaces where the geometry is close to $\ell_{2}$ as measured by a certain uniform comparison of the distance functions. The orthoplex space $\left(\mathbb{R}^{d}, \ell_{1}\right)$ has a $d / 2$-dimensional slice that is near-flat in this sense, whereas the largest such slice of the cube space $\left(\mathbb{R}^{d}, \ell_{\infty}\right)$ is $\log d$-dimensional. (See Ball [2] for a treatment of both almost spherical sections and volume ratio.) Yet at large scale $\mathbb{Z}^{d}$ with various generating sets can look like any of these; these observations should make it clear that the change of generators can make the free abelian group $\mathbb{Z}^{d}$ qualitatively quite different from Euclidean space.

Finally, let us consider the relationship of the sprawl to the Mahler Conjecture. For a convex, centrally symmetric body $\Omega$, define its polar body by

$$
\Omega^{\circ}:=\left\{x \in \mathbb{R}^{d}: \mathrm{x} \cdot \mathrm{y} \leq 1 \quad \forall \mathrm{y} \in \Omega\right\} .
$$

Thus for instance, the sphere is its own polar body in every dimension, $\left(\Omega^{\circ}\right)^{\circ}=\Omega$ and $\left(\text { Orth }_{d}\right)^{\circ}=$ Cube $_{d}$. The Mahler volume of $\Omega$ is defined to be

$$
M(\Omega)=\operatorname{Vol}(\Omega) \cdot \operatorname{Vol}\left(\Omega^{\circ}\right) .
$$

Let us also say that for any perimeter $L$, we write $M(L)$ for the Mahler volume of the convex hull of $L$, to unify notation. Just as for the sprawl, this is a statistic that is continuous in $\Omega$ and invariant under linear transformations. Mahler conjectured in 1939 that the extremes in every dimension were realized by the sphere and the cube, which would make $M$ yet another affine isoperimetric invariant that can be thought of as measuring "roundness versus pointiness" of a convex body. Santaló proved in 1949 that the spheres did indeed realize the upper bound on Mahler volume, but the 
lower bound is still an open problem in dimension $d>2$, despite some interesting recent progress by Kuperberg and others. Kuperberg gave a lower bound for Mahler volume of an arbitrary $\Omega$ as a multiple of the Mahler volume of the cube by a constant depending on the dimension; the constant is described as "a monotonic factor that begins at $4 / \pi$ and converges to $\sqrt{2}$ " [19, Corollary 1.6]. Comparing his formula to Example 11 above allows us to rewrite his result as

$$
M(\Omega) \geq\left(\frac{\pi}{4}\right)^{d} \cdot E\left(\text { Sphere }_{d}\right) \cdot M\left(\text { Cube }_{d}\right),
$$

so the sprawl makes a surprise appearance in the best known bound on the Mahler volume from below. Thus we hope that this study that began in finitely generated groups has produced an interesting object of consideration in convex geometry.

\section{References}

[1] J Arias-de-Reyna, K Ball, R Villa, Concentration of the distance in finite-dimensional normed spaces, Mathematika 45 (1998) 245-252 MR1695717

[2] K Ball, An elementary introduction to modern convex geometry, from: "Flavors of geometry", Math. Sci. Res. Inst. Publ. 31, Cambridge Univ. Press (1997) 1-58 MR1491097

[3] D Y Burago, Periodic metrics, from: "Representation theory and dynamical systems", Adv. Soviet Math. 9, Amer. Math. Soc., Providence, RI (1992) 205-210 MR1166203

[4] J W Cannon, Almost convex groups, Geom. Dedicata 22 (1987) 197-210 MR877210

[5] S Cleary, J Taback, Thompson's group F is not almost convex, J. Algebra 270 (2003) 133-149 MR2016653

[6] M Coornaert, Mesures de Patterson-Sullivan sur le bord d'un espace hyperbolique au sens de Gromov, Pacific J. Math. 159 (1993) 241-270 MR1214072

[7] S Dowdall, M Duchin, H Masur, Statistical hyperbolicity in Teichmüller space arXiv: 1108.5416

[8] S Dowdall, M Duchin, H Masur, Spheres in the curve complex arXiv:1109.6338

[9] M Duchin, S Lelièvre, C Mooney, The geometry of spheres in free abelian groups arXiv: 1004.0053

[10] M Duchin, S Lelièvre, C Mooney, The sprawl conjecture for convex bodies, Preprint

[11] M Duchin, C Mooney, Fine asymptotic geometry in the Heisenberg group arXiv: 1106.5276

[12] M Elder, S Hermiller, Minimal almost convexity, J. Group Theory 8 (2005) 239-266 MR2126733 
[13] A Eskin, D Fisher, K Whyte, Quasi-isometries and rigidity of solvable groups, Pure Appl. Math. Q. 3 (2007) 927-947 MR2402598

[14] S M Gersten, Quadratic divergence of geodesics in CAT(0) spaces, Geom. Funct. Anal. 4 (1994) 37-51 MR1254309

[15] S M Gersten, Divergence in 3-manifold groups, Geom. Funct. Anal. 4 (1994) 633-647 MR1302334

[16] E Gluskin, V Milman, Randomizing properties of convex high-dimensional bodies and some geometric inequalities, C. R. Math. Acad. Sci. Paris 334 (2002) 875-879 MR1909931

[17] S Hermiller, J Meier, Measuring the tameness of almost convex groups, Trans. Amer. Math. Soc. 353 (2001) 943-962 MR1804409

[18] M Kapovich, B Leeb, 3-manifold groups and nonpositive curvature, Geom. Funct. Anal. 8 (1998) 841-852 MR1650098

[19] G Kuperberg, From the Mahler conjecture to Gauss linking integrals, Geom. Funct. Anal. 18 (2008) 870-892 MR2438998

[20] E Lutwak, Selected affine isoperimetric inequalities, from: "Handbook of convex geometry, Vol. A, B”, North-Holland, Amsterdam (1993) 151-176 MR1242979

[21] Y Ollivier, Ricci curvature of Markov chains on metric spaces, J. Funct. Anal. 256 (2009) 810-864 MR2484937

[22] Y Ollivier, C Villani, A curved Brunn-Minkowski inequality on the discrete hypercube arXiv: 1011.4779

[23] W Woess, Lamplighters, Diestel-Leader graphs, random walks, and harmonic functions, Combin. Probab. Comput. 14 (2005) 415-433 MR2138121

Department of Mathematics, Tufts University

Medford 02155, USA

Laboratoire de mathématique d'Orsay, Université Paris-Sud (Paris 11)

91405 Orsay cedex, France

Department of Mathematics, Bradley University

Peoria, IL 61625, USA

moon.duchin@tufts.edu, samuel.lelievre@math.u-psud.fr,

cpmooney@bradley . edu

http://mduchin.math.tufts.edu, http://www.math.u-psud.fr/ lelievre/, http://hilltop.bradley.edu/ cpmooney

Received: 15 March $2011 \quad$ Revised: 6 September 2011 